\title{
Spectral analysis in thin tubes with axial heterogeneities
}

\author{
Rita Ferreira*, M. Luísa Mascarenhas**, and Andrey Piatnitski \\ (Communicated by Hugo Beirão da Veiga and José Francisco Rodrigues)
}

Dedicated to Professor João Paulo de Carvalho Dias on the occasion of his 70th birthday

\begin{abstract}
In this paper, we present the 3D-1D asymptotic analysis of the Dirichlet spectral problem associated with an elliptic operator with axial periodic heterogeneities. We extend to the $3 \mathrm{D}-1 \mathrm{D}$ case previous $3 \mathrm{D}-2 \mathrm{D}$ results (see [10]) and we analyze the special case where the scale of thickness is much smaller than the scale of the heterogeneities and the planar coefficient has a unique global minimum in the periodic cell. These results are of great relevance in the comprehension of the wave propagation in nanowires showing axial heterogeneities (see [17]).
\end{abstract}

Mathematics Subject Classification (primary; secondary): 35P20, 35B27; 49R05, 47A75, 81Q15

Keywords: Spectral analysis, dimension reduction, homogenization, quantum waveguides

\section{Introduction and main results}

The motivation to manipulate matter on the nanometer scale arises not only from the emergence of novel behaviors at small length-scales, but also from the appeal of engineering material properties to building up from the nanoscale (see [5], [17], [18], [19], [21], [24], [25], and references therein).

A true nanotechnology based on materials built from the bottom up requires a rigorous mathematical analysis to obtain the corresponding effective behavior. With a diameter of the order of a nanometer, nanowires are almost one-

\footnotetext{
*R. Ferreira was partially supported by the Fundação para a Ciência e a Tecnologia (Portuguese Foundation for Science and Technology) through SFRH/BPD/81442/2011 and by the KAUST SRI, Center for Uncertainty Quantification in Computational Science and Engineering.

**The research of M. L. Mascarenhas was funded by UID/MAT/00297/2013. M. L. Mascarenhas thanks the warm hospitality of Narvik University, where the final part of this work was undertaken.
} 
dimensional objects, which have special physical and chemical properties different from those of their bulk counterparts. Also composite thin structures presenting fast periodic oscillations, localized curvature or torsion, are interesting features. The study of their interactions is of the highest importance in applications.

Previous mathematical analysis has been performed concerning the relation between the effects of curvature, torsion, or transversal heterogeneities in wave propagation through thin tubes (see, for instance, [2], [3], [4], [6], [8], [11], [15], [16]).

The goal of this work is to analyze the interaction between thickness and axial periodic heterogeneities in terms of their effect on electron transport through a thin tube-shaped domain. We present a sharp analysis of the corresponding levels of energy. Effective one-dimensional limit problems are derived explicitly by the use of analytical tools. Our rigorous analysis can serve as a basis for numerical simulations of sophisticated devices involving nanowires presenting axial heterogeneities.

Ballistic transport in modulated semiconductor devices, such as nanowire heterostructures, is governed by the effective-mass Schrödinger equation

$$
-\frac{\hbar^{2}}{2} \operatorname{div}\left(\frac{1}{m(z)} \nabla \psi(z)\right)+V(z) \psi(z)=E \psi(z), \quad z \in \mathbb{R}^{3},
$$

where $V$ is a sharp potential that is zero inside the confinement imposed by the device geometry and infinite outside. The quantities $\psi$ and $E$ stand for the wave function and the energy, respectively. The spatial position-dependence of the effective mass $m$ allows to model material consisting of different components. In what follows, we use a notation that is more common in the mathematical literature and rewrite (1.1) as an elliptic Dirichlet eigenvalue problem of the form

$$
\left\{\begin{array}{l}
-\operatorname{div}\left(A_{\varepsilon} \nabla \tilde{v}_{\varepsilon}^{\delta}\right)=\lambda_{\varepsilon}^{\delta} \tilde{v}_{\varepsilon}^{\delta}, \quad \text { in } \delta \omega \times I, \\
\tilde{v}_{\varepsilon}^{\delta} \in H_{0}^{1}(\delta \omega \times I),
\end{array}\right.
$$

where $\omega$ is an open bounded domain in $\mathbb{R}^{2}, I$ is the interval $(0, L), L>0$, and where $\varepsilon$ and $\delta$ are small parameters: $\delta$ represents the thickness of the thin domain and $\varepsilon$ the length scale of the heterogeneities. These heterogeneities are encoded in a $3 \times 3$ matrix $A_{\varepsilon}$, which only depends on the third variable and is defined by

$$
A_{\varepsilon}\left(x_{3}\right):=A\left(\frac{x_{3}}{\varepsilon}\right), \quad \text { a.e. } x_{3} \in \mathbb{R} .
$$

As suggested by the effective-mass Schrödinger equation, we consider the particular case of a diagonal and $(0,1)$-periodic matrix $A=\left(a_{i j}\right)_{1 \leq i, j \leq 3} \in\left[L^{\infty}(\mathbb{R})\right]^{3 \times 3}$ such that: 


$$
\begin{aligned}
& a_{\alpha \beta}(y)=b(y) \delta_{\alpha, \beta}, \quad a_{33}(y)=a(y) \\
& \eta \leq a(y), b(y) \leq \zeta
\end{aligned}
$$

a.e. $y \in Y:=[0,1]$, and for some $0<\eta<\zeta$.

The general case of a non-diagonal matrix requires a different treatment and will be the subject of a separate paper.

For each $\varepsilon>0$, we set $a_{\varepsilon}\left(x_{3}\right):=a\left(x_{3} / \varepsilon\right)$ and $b_{\varepsilon}\left(x_{3}\right):=b\left(x_{3} / \varepsilon\right)$.

This paper deals with the asymptotic behavior of spectral problem (1.2) as both positive parameters $\varepsilon$ and $\delta$ tend to zero. The proofs and statements of Theorem 1.1 concerning the case $\varepsilon \approx \delta$, Theorem 1.2 concerning the case $\varepsilon \ll \delta$, and Theorems 1.4 and 1.5 regarding the case $\varepsilon \gg \delta$, are rather similar to those obtained in [10] (see also [9]) for the 3D-2D setting. For the sake of completeness we state these results, but we skip the details of their proofs. Here, the main result is Theorem 1.9, where we consider the case $\varepsilon \gg \delta$ under different assumptions from those in $[10]$.

In Section 2, we present the principal auxiliary results, which give us the general tools for the proofs of the statements stated below and, in Section 3, we provide a detailed proof of Theorem 1.9.

Considering the quadratic energy $\tilde{E}_{\varepsilon}^{\delta}: L^{2}(\delta \omega \times I) \rightarrow[0,+\infty]$ associated with the self-adjoint operator $-\operatorname{div}\left(A_{\varepsilon} \nabla \cdot\right)$ from $L^{2}(\delta \omega \times I)$ into itself, we obtain

$$
\tilde{E}_{\varepsilon}^{\delta}(\tilde{v}):= \begin{cases}\int_{\delta \omega \times I} A_{\varepsilon}\left(x_{3}^{\delta}\right) \nabla \tilde{v}\left(x^{\delta}\right) \nabla \tilde{v}\left(x^{\delta}\right) \mathrm{d} x^{\delta}, & \text { if } \tilde{v} \in H_{0}^{1}(\delta \omega \times I) \\ +\infty, & \text { otherwise. }\end{cases}
$$

To proceed with our analysis of the asymptotic behavior of problem (1.2) as $\delta$ and $\varepsilon$ go to zero, the first step is to perform a change of variables and a rescaling, in order to transform the studied problem into an equivalent problem defined in the fixed domain $\omega \times I$.

Let $\bar{x}$ stand for $\left(x_{1}, x_{2}\right)$. To each point $x^{\delta}=\left(\bar{x}^{\delta}, x_{3}^{\delta}\right) \in \delta \omega \times I$ we associate the point $x=\left(\bar{x}, x_{3}\right)=\left(\delta^{-1} \bar{x}^{\delta}, x_{3}^{\delta}\right) \in \omega \times I$, and we define $v \in H_{0}^{1}(\omega \times I)$ by $v(x):=\tilde{v}\left(x^{\delta}\right)$ whenever $\tilde{v} \in H_{0}^{1}(\delta \omega \times I)$. Accordingly, we rescale the energy in (1.5) by dividing it by $\delta^{2}$, so that the new energy becomes $E_{\varepsilon}^{\delta}: H_{0}^{1}(\omega \times I) \rightarrow \mathbb{R}$,

$$
E_{\varepsilon}^{\delta}(v):=\int_{\omega \times I} a_{\varepsilon}\left(x_{3}\right)\left|\partial_{3} v(x)\right|^{2}+\frac{b_{\varepsilon}\left(x_{3}\right)}{\delta^{2}}|\bar{\nabla} v(x)|^{2} \mathrm{~d} x,
$$

where $\bar{\nabla}$ stands for $\left(\partial / \partial x_{1}, \partial / \partial x_{2}\right), \bar{\Delta}$ for $\partial^{2} / \partial x_{1}^{2}+\partial^{2} / \partial x_{2}^{2}$, and $\partial_{3}$ for $\partial / \partial x_{3}$.

The rescaled spectral problem is then

$$
\left\{\begin{array}{l}
-\partial_{3}\left(a_{\varepsilon} \partial_{3} v\right)-\frac{b_{\varepsilon}}{\delta^{2}} \bar{\Delta} v=\lambda v, \quad \text { in } \omega \times I, \\
v \in H_{0}^{1}(\omega \times I)
\end{array}\right.
$$


We stress that problems (1.2) and (1.6) are equivalent.

Before stating the results, let us introduce some notations. Since we are interested in the cases $\varepsilon \approx \delta, \varepsilon \ll \delta$, and $\varepsilon \gg \delta$, we consider $\delta=\varepsilon^{\tau}$ for each $\tau \in(0,+\infty)$.

We introduce the first normalized eigenpair $\left(\mu_{\varepsilon, 0}^{\tau}, \phi_{\varepsilon, 0}^{\tau}\right)$ for the one-dimensional periodic spectral problem

$$
\left\{\begin{array}{l}
-\varepsilon^{2(\tau-1)}\left(a\left(\phi_{\varepsilon}^{\tau}\right)^{\prime}\right)^{\prime}+b \rho_{0} \phi_{\varepsilon}^{\tau}=\mu_{\varepsilon}^{\tau} \phi_{\varepsilon}^{\tau}, \quad \text { a.e. in } Y, \\
\phi_{\varepsilon}^{\tau} \in H_{\#}^{1}(Y),
\end{array}\right.
$$

where $\rho_{0}$ is the first eigenvalue of the following bi-dimensional spectral problem in $\omega$ :

$$
\left\{\begin{array}{l}
-\Delta \theta=\rho \theta, \quad \text { a.e. in } \omega, \\
\theta \in H_{0}^{1}(\omega) .
\end{array}\right.
$$

We recall that $C_{\#}^{\infty}(Y)$ (resp. $\left.C_{\#}(Y)\right)$ represents the subspace of $C^{\infty}(\mathbb{R})$ (resp. $C(\mathbb{R}))$ of $Y$-periodic functions, and $H_{\#}^{1}(Y)$ is the closure of $C_{\#}^{\infty}(Y)$ with respect to the $H^{1}(Y)$-norm. Furthermore, the eigenvalues $\mu_{\varepsilon, 0}^{\tau}$ and $\rho_{0}$ are positive, and simple, and the associated normalized eigenfunctions $\phi_{\varepsilon, 0}^{\tau}$ and $\theta_{0}$ may be chosen to be strictly positive functions.

Since in the case $\varepsilon \approx \delta(\tau=1)$ problem (1.7) does not depend on $\varepsilon$, we write $\left(\mu_{0}, \phi_{0}\right)$ to denote its normalized first eigenpair.

Theorem $1.1(\varepsilon \approx \delta, \tau=1)$. Under hypothesis (1.3) and (1.4), let $\left(\lambda_{\varepsilon, k}, v_{\varepsilon, k}\right)$ be the sequence of normalized eigenpairs (with repetitions, according to multiplicity) to problem (1.6) with $\varepsilon=\delta$. Let $\left(v_{k}, \varphi_{k}\right)$ be the sequence of normalized eigenpairs to the following one-dimensional homogenized spectral problem (whose eigenvalues are simple)

$$
\left\{\begin{array}{l}
-\left(\tilde{a}^{h} \varphi^{\prime}\right)^{\prime}=v \varphi, \text { a.e. in } I, \\
\varphi \in H_{0}^{1}(I),
\end{array}\right.
$$

where $|Y|$ stands for the length of $Y$, and

$$
\tilde{a}^{h}:=\left(\frac{1}{|Y|} \int_{Y} \frac{1}{\left[\phi_{0}(y)\right]^{2} a(y)} \mathrm{d} y\right)^{-1}
$$

is the homogenized coefficient of divergence form operators with coefficients

$$
\tilde{a}_{\varepsilon}\left(x_{3}\right):=\left[\phi_{0}\left(\frac{x_{3}}{\varepsilon}\right)\right]^{2} a\left(\frac{x_{3}}{\varepsilon}\right) .
$$


Then, for any $k \in \mathbb{N}_{0}$,

$$
\lambda_{\varepsilon, k}=\frac{\mu_{0}}{\varepsilon^{2}}+v_{\varepsilon, k}, \quad v_{\varepsilon, k}\left(\bar{x}, x_{3}\right)=\phi_{0}\left(\frac{x_{3}}{\varepsilon}\right) u_{\varepsilon, k}\left(\bar{x}, x_{3}\right),
$$

a.e. $\left(\bar{x}, x_{3}\right) \in \omega \times I$, where as $\varepsilon \rightarrow 0^{+}, v_{\varepsilon, k} \rightarrow v_{k}$ and $u_{\varepsilon, k} \rightarrow u_{k}$ weakly in $H_{0}^{1}(\omega \times I)$. Here, $u_{k}$ is the product of $\theta_{0}$ (see (1.8)) with an eigenfunction associated with $v_{k}$ (see (1.9)). Conversely, any eigenfunction $u_{k}=\varphi_{k} \theta_{0}$ is the weak limit of a particular sequence of the form

$$
u_{\varepsilon, k}=\left[\phi_{0}\left(\frac{x_{3}}{\varepsilon}\right)\right]^{-1} v_{\varepsilon, k}
$$

Next, we describe the spectrum in the case $\varepsilon \ll \delta$. For $j \in \mathbb{N}_{0}$, define

$$
\gamma_{j}:=\rho_{0} \int_{Y} b(y) \psi_{j}(y) \mathrm{d} y,
$$

where $\psi_{0} \equiv 1$ in $Y$ and, for $j \geq 1, \psi_{j}$ are the solutions of the recurrent problems in $H_{\#}^{1}(Y)$

$$
-\left(a(y) \psi_{j}^{\prime}\right)^{\prime}=-b(y) \rho_{0} \psi_{j-1}+\sum_{\ell=0}^{j-1} \gamma_{\ell} \psi_{j-1-\ell}, \quad \int_{Y} \psi_{j}(y) \mathrm{d} y=0 .
$$

Theorem $1.2(\varepsilon \ll \delta, \tau<1)$. Suppose that hypotheses (1.3) and (1.4) are satisfied. Let $\left(\lambda_{\varepsilon, k}, v_{\varepsilon, k}\right)$ be the sequence of normalized eigenpairs (with repetitions, according to multiplicity) associated with problem (1.6) for $\delta=\varepsilon^{\tau}$ and some $\tau \in(0,1)$. Let $i \in \mathbb{N}$ be such that $\frac{i-1}{i}<\tau \leq \frac{i}{i+1}$, and let $\left(\mu_{\varepsilon, 0}^{\tau}, \phi_{\varepsilon, 0}^{\tau}\right)$ be the normalized first eigenpair of (1.7). Finally, let $\left(v_{k}, \varphi_{k}\right)$ be the sequence of normalized eigenpairs associated with the following homogenized spectral problem

$$
\left\{\begin{array}{l}
-\left(a^{h} \varphi^{\prime}\right)^{\prime}=v \varphi, \quad \text { a.e. in } I, \\
\varphi \in H_{0}^{1}(I),
\end{array}\right.
$$

where

$$
a^{h}:=\left(\frac{1}{|Y|} \int_{Y} \frac{1}{a(y)} \mathrm{d} y\right)^{-1}
$$

Then, as $\varepsilon \rightarrow 0^{+}, \mu_{\varepsilon, 0}^{\tau} \rightarrow \rho_{0} \int_{Y} b(y) \mathrm{d} y=\gamma_{0}, \phi_{\varepsilon, 0}^{\tau}\left(x_{3} / \varepsilon\right) \rightarrow 1=\psi_{0}$ uniformly in $I$, and

$$
\lambda_{\varepsilon, k}=\sum_{j=0}^{i} \frac{\gamma_{j}}{\varepsilon^{\tau(2 j+2)-2 j}}+r_{\varepsilon}^{\tau}+v_{\varepsilon, k}^{\tau}, \quad v_{\varepsilon, k}\left(\bar{x}, x_{3}\right)=\phi_{\varepsilon, 0}^{\tau}\left(\frac{x_{3}}{\varepsilon}\right) u_{\varepsilon, k}\left(\bar{x}, x_{3}\right)
$$


a.e. $\left(\bar{x}, x_{3}\right) \in \omega \times I$, where $v_{\varepsilon, k}^{\tau}, u_{\varepsilon, k}$, and $r_{\varepsilon}^{\tau}$ satisfy, as $\varepsilon \rightarrow 0^{+}: r_{\varepsilon}^{\tau} \rightarrow 0, v_{\varepsilon, k}^{\tau} \rightarrow v_{k}$, and $u_{\varepsilon, k} \rightarrow u_{k}$ weakly in $H_{0}^{1}(\omega \times I)$. Here, $u_{k}$ is the product of the eigenfunction associated with $v_{k}$ and $\theta_{0}$. Conversely, any eigenfunction $u_{k}=\varphi_{k} \theta_{0}$ is the weak limit of a sequence of the form

$$
u_{\varepsilon, k}=\left[\phi_{\varepsilon, 0}^{\tau}\left(\frac{x_{3}}{\varepsilon}\right)\right]^{-1} v_{\varepsilon, k} .
$$

Remark 1.3. In the present 3D-1D case, no further regularity assumptions on the coefficients are needed. This contrasts with the 3D-2D case, where we have to impose uniform Lipschitz continuity on the planar coefficients in order to ensure the continuity of the principal cell mode (see [10]).

The case $\varepsilon \gg \delta(\tau>1)$ is more delicate to handle due to the degeneracy of the corresponding problem (1.7). Indeed, in that case, the asymptotic behavior of $\mu_{\varepsilon, 0}^{\tau}$ depends essentially on the behavior of the potential $a$ (see, for instance, [13], [14]).

Next, we state a general theorem for the case $\varepsilon \gg \delta$, which provides a characterization of the limit behavior of spectrum in the Hausdorff sense. Then we consider two specific cases, in which we are able to characterize the asymptotic behavior of the eigenvalues. It is interesting to notice the differences between those two cases.

Theorem $1.4(\varepsilon \gg \delta, \tau>1)$. Assume hypotheses (1.3)-(1.4) and, in addition, that $b$ attains a minimum value, $b_{\min }$, at some $y_{0} \in Y$ such that $a$ and $b$ are continuous on some neighborhood of $y_{0}$. Then, denoting the spectrum of our initial problem by $\sigma_{\varepsilon}$, he have

$$
\lim _{\varepsilon \rightarrow 0^{+}}\left(\varepsilon^{2 \tau} \sigma_{\varepsilon}\right)=\left[b_{\min } \rho_{0},+\infty\right],
$$

where the limit in (1.11) is to be understood in the sense of Hausdorff, that is, $\left[b_{\min } \rho_{0},+\infty\right]$ is the set of all cluster points of sequences $\left\{\lambda_{\varepsilon}\right\}_{\varepsilon>0}, \lambda_{\varepsilon} \in \varepsilon^{2 \tau} \sigma_{\varepsilon}$.

Next we proceed with the first particular case.

Theorem $1.5(\varepsilon \gg \delta, \tau>1)$. Let hypotheses (1.3)-(1.4) hold, and assume that a is a smooth function and that there exists a nonempty open interval $Q, Q \subset \subset Y$, such that $b=b_{\min }$ in $Q$. Assume, furthermore, that on $Y \backslash Q$ the function $b$ is smooth and satisfies the inequality $b \geq b_{\min }+\kappa$ for some $\kappa>0$. Let $\left(v_{0}, q_{0}\right)$ be the normalized first eigenpair of the one-dimensional spectral problem on $Q$

$$
\left\{\begin{array}{l}
-\left(a q^{\prime}\right)^{\prime}=v q, \quad \text { a.e. in } Q \\
q \in H_{0}^{1}(Q) .
\end{array}\right.
$$


Let $i$ be an integer such that $i \geq \frac{2}{\tau-1}$, and let $\left(\mu_{\varepsilon, 0}^{\tau}, \phi_{\varepsilon, 0}^{\tau}\right)$ be the normalized first eigenpair of (1.7). Then $\mu_{\varepsilon, 0}^{\tau} \rightarrow b_{\min } \rho_{0}, \phi_{\varepsilon, 0}^{\tau} \rightarrow q_{0}$ weakly in $H_{\#}^{1}(Y)$ as $\varepsilon \rightarrow 0^{+}$, where we identify $q_{0}$ with its extension by zero to the whole $Y$. Moreover, for any $k \in \mathbb{N}_{0}$,

$$
\lambda_{\varepsilon, k}=\frac{b_{\min } \rho_{0}}{\varepsilon^{2 \tau}}+\frac{v_{0}}{\varepsilon^{2}}+\varepsilon^{\tau-3} \mu_{1}+\cdots+\varepsilon^{i(\tau-1)-2} \mu_{i}+\rho_{\varepsilon, i}^{\tau}+v_{\varepsilon, k}^{\tau},
$$

where $\mu_{j}, j \in\{1, \ldots, i\}$, are well-determined constants, $\rho_{\varepsilon, i}^{\tau}$ satisfies $\left|\rho_{\varepsilon, i}^{\tau}\right| \leq$ $C \varepsilon^{(i+1 / 2)(\tau-1)-2}$, for some constant $C$ independent of $\varepsilon$, and $v_{\varepsilon, k}^{\tau} \rightarrow 0$, as $\varepsilon \rightarrow 0^{+}$.

Remark 1.6. Theorem 1.5 is valid under weaker regularity hypotheses on the coefficients. In fact, as it becomes clear within the proof (see [10]), it suffices to assume that $a$ is a $C^{i+2}$ function and that on $Y \backslash Q, b$ is also a $C^{i+2}$ function, where $i$ is the smallest natural number satisfying $i \geq \frac{2}{\tau-1}$. In fact, the smaller $\tau-1>0$ is, the more regularity is required for the coefficients.

Remark 1.7. Hypotheses of Theorem 1.5 cover the important case where $b$ oscillates between two different values, but rule out the case where $b$ is constant. Nevertheless, it is easy to see that under the general hypotheses stated at the beginning of this section, if $b$ is constant, then for any $\tau \in(0,+\infty), \mu_{\varepsilon, 0}^{\tau} \equiv b \rho_{0}$ and $\phi_{\varepsilon, 0}^{\tau} \equiv 1$. Moreover, if $\left(\lambda_{\varepsilon, k}, v_{\varepsilon, k}\right)$ is the sequence of normalized eigenpairs associated to problem (1.6) for $\delta=\varepsilon^{\tau}$, then

$$
\lambda_{\varepsilon, k}=\frac{b \rho_{0}}{\varepsilon^{2 \tau}}+v_{\varepsilon, k},
$$

where $v_{\varepsilon, k} \rightarrow v_{k}$ and $v_{\varepsilon, k} \rightarrow v_{k}=\varphi_{k} \theta_{0}$ weakly in $H_{0}^{1}(\omega \times I)$ as $\varepsilon \rightarrow 0^{+},\left(v_{k}, \varphi_{k}\right)$ being associated with (1.10).

Remark 1.8. Theorem 1.5 deals with the case of $b$ attaining its minimum in the interval $Q$ and jumping up at the endpoints of this interval so that $b>b_{\min }+\kappa$ on $Y \backslash Q$ for some $\kappa>0$. One can assume instead that $b$ is continuous in $Y$, smooth in $Y \backslash \bar{Q}, b=b_{\min }$ in $Q$, and $b>b_{\min }$ in $Y \backslash \bar{Q}$. In this case, the first two terms in (1.12) remain unchanged while the lower order terms should be introduced in a different way. The structure of these terms depends on the behavior of $b$ in the vicinity of the endpoints of $Q$. If on both sides of $Q$ the linear terms of the Taylor series of $\left(b-b_{\min }\right)$ do not vanish, then

$$
\lambda_{\varepsilon, k}=\frac{b_{\min } \rho_{0}}{\varepsilon^{2 \tau}}+\frac{\nu_{0}}{\varepsilon^{2}}+\sum_{i=1}^{N_{0}} \varepsilon^{(2 i(\tau-1)) / 3-2} \mu_{i}+\rho_{\varepsilon, i}^{\tau}+v_{\varepsilon, k}^{\tau}, \quad N_{0}=\left[2 \frac{3}{2(\tau-1)}\right]
$$


here the symbol $[\cdot]$ stands for the integer part. If all the terms of the referred Taylor series up to order $(k-1)$ vanish and the terms of order $k$ do not, then

$$
\lambda_{\varepsilon, k}=\frac{b_{\min } \rho_{0}}{\varepsilon^{2 \tau}}+\frac{v_{0}}{\varepsilon^{2}}+\sum_{i=1}^{N_{0}} \varepsilon^{(2 i(\tau-1)) /(k+2)-2} \mu_{i}+\rho_{\varepsilon, i}^{\tau}+v_{\varepsilon, k}^{\tau}, \quad N_{0}=\left[2 \frac{k+2}{2(\tau-1)}\right] .
$$

The method of determining $\mu_{i}$ relies on constructing boundary layer correctors in the neighborhood of the endpoints of $Q$. The case when the degeneration of $\left(b-b_{\min }\right)$ at the endpoints of $Q$ is not of the same order can also be treated.

We now proceed with the case of a unique global minimum of $b$. We stress the different behavior of the spectrum compared with the previous case.

Theorem $1.9(\varepsilon \gg \delta, \tau>1)$. Under hypotheses (1.3)-(1.4), assume, in addition, that $b$ is continuous and attains a global minimum $b_{\min }$ at a unique point $y_{0} \in Y$. Suppose that $a$ and $b$ are smooth in a neighborhood of $y_{0}$ and that, moreover, $b^{\prime \prime}\left(y_{0}\right) \neq 0$. Let $\sigma_{\varepsilon}:=\left\{\lambda_{\varepsilon, k} \in \mathbb{R}^{+}: k \geq 0\right\}$ be the spectrum of problem (1.6) for $\delta=\varepsilon^{\tau}$ and $\tau \in(1,+\infty)$, and let $\left(v_{0}, \psi_{0}\right)$ be the first normalized eigenpair of the following quantum harmonic oscillator

$$
\left\{\begin{array}{l}
-a\left(y_{0}\right) \psi^{\prime \prime}+\frac{b^{\prime \prime}\left(y_{0}\right) \rho_{0}}{2} t^{2} \psi=v \psi, \quad \text { a.e. in } \mathbb{R}, \\
\psi \in H^{1}(\mathbb{R}) .
\end{array}\right.
$$

Then, for $i \in \mathbb{N}, i>\frac{3 \tau+1}{\tau-1}$,

$$
\lambda_{\varepsilon, k}=\frac{b_{\min } \rho_{0}}{\varepsilon^{2 \tau}}+\frac{v_{0}}{\varepsilon^{\tau+1}}+\frac{\mu_{3}}{\varepsilon^{(\tau+3) / 2}}+\cdots+\varepsilon^{((i+2) / 2)(\tau-1)-2 \tau} \mu_{i+2}+\rho_{\varepsilon, i}^{\tau}+v_{\varepsilon, k}^{\tau},
$$

where $\rho_{0}$ is the first eigenvalue of problem (1.8), $\mu_{j}, j \in\{3, \ldots, i+2\}$, are welldetermined constants, $\rho_{\varepsilon, i}^{\tau}$ satisfies $\left|\rho_{\varepsilon, i}^{\tau}\right| \leq C \varepsilon^{((i+1)(\tau-1)) / 2-2 \tau}$, for a constant $C$ independent of $\varepsilon$, and $v_{\varepsilon, k}^{\tau} \rightarrow 0$, as $\varepsilon \rightarrow 0^{+}$.

Remark 1.10. Observe that in the case $\tau>1$, due to the singular structure of cell problem (1.7), the coefficients $\mu_{j}, j \in\{3, \ldots, i+2\}$, in (1.14) do not depend on $k$.

\section{Auxiliary results}

In this section we present the auxiliary results that play an important role in the sequel. 
In the first statement we make a dimension reduction in eigenvalue problem (1.6).

Proposition 2.1. Let $a$ and $b$ be two measurable functions in $\mathbb{R}$ satisfying almost everywhere bounds (1.4), with $0<\zeta<\eta$. For each $n \geq 0$, let $\left\{\lambda_{k}^{(n)}\right\}_{k \geq 0}$ be the spectrum of the following eigenvalue problem:

$$
\left\{\begin{array}{l}
-\left(a \psi^{\prime}\right)^{\prime}+b \rho_{n} \psi=\lambda \psi, \quad \text { a.e. in } I, \\
\varphi \in H_{0}^{1}(I),
\end{array}\right.
$$

where $\rho_{n}$ is the $n$-th eigenvalue in (1.8). Then the set $\left\{\lambda_{k}^{(n)}\right\}_{k, n \geq 0}$ coincides with the spectrum of the three-dimensional spectral problem

$$
\left\{\begin{array}{l}
-\partial_{3}\left(a \partial_{3} v\right)-b \bar{\Delta} v=\lambda v, \quad \text { a.e. in } \omega \times I, \\
v \in H_{0}^{1}(\omega \times I) .
\end{array}\right.
$$

In particular, $\lambda_{0}=\lambda_{0}^{(0)}$.

Proof. Let $\left(\rho_{n}, \theta_{n}\right)$ be the normalized sequence of eigenpairs of problem (1.8) and $\left(\lambda_{k}^{(n)}, \psi_{k}^{(n)}\right)$ the sequence of normalized eigenpairs of problem (2.1) (with repetitions according to multiplicity). Then, it can be checked that

1) The family of functions $\left\{v_{k}^{(n)}=\psi_{k}^{(n)}\left(x_{3}\right) \theta_{n}(\bar{x}), n \geq 0, k \geq 0\right\}$ is an orthonormal basis in $L^{2}(\omega \times I)$;

2) $\left(\lambda_{k}^{(n)}, v_{k}^{(n)}\right), k, n \in \mathbb{N}_{0}$, are eigenpairs of (2.2).

The domain of the operator $-\partial_{3}\left(a \partial_{3}\right)-b \bar{\Delta}$ is a linear subset of $H_{0}^{1}(\omega \times I)$ which is dense in $L^{2}(\omega \times I)$. Since this operator is coercive and self-adjoint in $L^{2}(\omega \times I)$ and has a compact resolvent, in view of 1) and 2) and using the Fredholm Theorem, we conclude that its spectrum coincides with $\left\{\lambda_{k}^{(n)}\right\}_{k, n \in \mathbb{N}_{0}}$. This completes the proof.

The second proposition regards a classical change of unknowns (c.f. [22]; see also [1]).

Proposition 2.2. For fixed $\tau, \varepsilon>0$, consider the functions $u$ and $v$ related by the formula

$$
v(x)=\phi_{\varepsilon, 0}^{\tau}\left(\frac{x_{3}}{\varepsilon}\right) u(x), \quad \text { a.e. } x=\left(\bar{x}, x_{3}\right) \in \omega \times I .
$$

Then $v \in H_{0}^{1}(\omega \times I)$ if and only if $u \in H_{0}^{1}(\omega \times I)$. Moreover, if $v \in H_{0}^{1}(\omega \times I)$, we have 


$$
\begin{gathered}
\int_{\omega \times I} a_{\varepsilon}\left(x_{3}\right)\left|\partial_{3} v(x)\right|^{2}+\frac{b_{\varepsilon}\left(x_{3}\right)}{\varepsilon^{2 \tau}} \rho_{0} v^{2}(x)-\frac{\mu_{\varepsilon, 0}^{\tau}}{\varepsilon^{2 \tau}} v^{2}(x) \mathrm{d} x \\
=\int_{\omega \times I} a_{\varepsilon}\left(x_{3}\right)\left[\phi_{\varepsilon, 0}^{\tau}\left(\frac{x_{3}}{\varepsilon}\right)\right]^{2}\left|\partial_{3} u(x)\right|^{2} \mathrm{~d} x,
\end{gathered}
$$

where $\left(\mu_{\varepsilon, 0}^{\tau}, \phi_{\varepsilon, 0}^{\tau}\right)$ is the first normalized eigenpair of the cell problem (1.7).

Remark 2.3. Applying Propositions 2.1 and 2.2 to the rescaled problem (1.6) with $\delta=\varepsilon^{\tau}$, we obtain that

$$
\lambda_{\varepsilon, k}^{(0)}=\frac{\mu_{\varepsilon, 0}^{\tau}}{\varepsilon^{2 \tau}}+v_{\varepsilon, k}^{\tau}
$$

where

$$
v_{\varepsilon, k}^{\tau}:=\inf _{S_{k}} \max _{\substack{\psi \in S_{k} \\\left\|\phi_{\varepsilon, 0}^{\tau}\left(\frac{\dot{\varepsilon}}{\varepsilon}\right) \psi\right\|_{L^{2}(I)}=1}}\left\{\int_{I}\left|\phi_{\varepsilon, 0}^{\tau}\left(\frac{x_{3}}{\varepsilon}\right)\right|^{2} a_{\varepsilon}\left(x_{3}\right)\left|\psi^{\prime}\left(x_{3}\right)\right|^{2} \mathrm{~d} x_{3}\right\},
$$

and the infimum is taken over all $(k+1)$-dimensional subspaces $S_{k}$ of $H_{0}^{1}(I)$.

Since $v_{\varepsilon, k}^{\tau}$ converges to $v_{k}$, as $\varepsilon \rightarrow 0^{+}$, for any $k \geq 0$ we have $\left|\lambda_{\varepsilon, k}^{(0)}-\frac{\mu_{\varepsilon, 0}^{\tau}}{\varepsilon^{2 \tau}}\right| \leq C_{k}$, if $\varepsilon$ is small enough. On the other hand, from the variational representation for the eigenvalues it easily follows that $\lambda_{\varepsilon, 0}^{(1)}-\lambda_{\varepsilon, 0}^{(0)} \geq \frac{\rho_{1}-\rho_{0}}{\varepsilon^{2 \tau}} b_{\min }$. Therefore, $\lambda_{\varepsilon, j}=\lambda_{\varepsilon, j}^{(0)}$ when $0 \leq j \leq k$, for sufficiently small $\varepsilon$.

Another important tool in our spectral analysis is the following proposition (see [23] and [20], Lemma III.1.1).

Proposition 2.4. Let $L: H \rightarrow H$ be a linear compact self-adjoint operator in a Hilbert space $H$, and suppose that $\lambda>0$ and $f \in H$ are such that $\|L f-\lambda f\|_{H} \leq \gamma$, for some constant $\gamma \in \mathbb{R}^{+}$. Then there exists an eigenvalue $\bar{\lambda}$ of $L$ such that $|\bar{\lambda}-\lambda| \leq \gamma\|f\|_{H}^{-1}$. Furthermore, for $d>\gamma\|f\|_{H}^{-1}$, there exists a $\bar{f},\|\bar{f}\|_{H}=\|f\|_{H}$, linear combination of eigenvectors associated with the eigenvalues lying in the interval $[\lambda-d, \lambda+d]$, and satisfying $\|\bar{f}-f\|_{H} \leq 2 d^{-1} \gamma$.

\section{Proof of Theorem 1.9}

As we have seen in the previous section, the asymptotic behavior of the eigenvalues and eigenmodes of the cell problem (1.7) is crucial for further considerations. The study of problem (1.7) relies on blow up analysis in the vicinity of $y_{0}$. 
We recall, then, some classical results about the quantum harmonic oscillator. Consider the Hamiltonian of the harmonic oscillator $\mathscr{A} \psi=-\psi^{\prime \prime}+v_{0}^{2} t^{2} \psi\left(v_{0}>0\right)$. For $\psi, \varphi \in \mathscr{D}(\mathbb{R})$, denote by $\langle$,$\rangle the duality between \mathscr{D}^{\prime}(\mathbb{R})$ and $\mathscr{D}(\mathbb{R})$. Then

$$
\langle\mathscr{A} \psi, \varphi\rangle=\int_{\mathbb{R}}\left(\psi^{\prime} \varphi^{\prime}+v_{0}^{2} t^{2} \psi \varphi\right) \mathrm{d} t=a(\psi, \varphi) .
$$

Defining $V$ as the completion of $\mathscr{D}(\mathbb{R})$ in the norm

$$
\|\psi\|_{V}:=\sqrt{\int_{\mathbb{R}}\left(\left|\psi^{\prime}\right|^{2}+t^{2}|\psi|^{2}\right) \mathrm{d} t}
$$

relation (3.1) still holds for $\psi, \varphi$ in the Hilbert space $V$. Furthermore, denoting $H=L^{2}(\mathbb{R})$, we have $V \subset H$, with compact embedding (see, for instance, [7], Propositions 25-26). Since $V$ is dense in $H$, and $a(\psi, \varphi)$ is coercive on $V$, the operator $\mathscr{A}$ is a positive self-adjoint operator in $L^{2}(\mathbb{R})$ with compact resolvent. So, the associated spectral problem:

$$
-\psi^{\prime \prime}+v_{0}^{2} t^{2} \psi=v \psi, \quad \psi \in V
$$

has its spectrum composed by simple eigenvalues, given by the sequence

$$
v_{n}=v_{0}(1+2 n), \quad n \geq 0 .
$$

Moreover, there exists an orthonormal basis of eigenfunctions in terms of Hermite polynomials as follows:

$$
\begin{aligned}
& \psi_{n}(t)=C_{n} e^{-v_{0}\left(t^{2} / 2\right)} H_{n}\left(t \sqrt{v_{0}}\right), \quad C_{n}:=\frac{\sqrt[4]{v_{0}}}{\left[2^{n} n ! \sqrt{\pi}\right]^{1 / 2}} \\
& H_{n}(t)=(-1)^{n} e^{t^{2}} \frac{d^{n}}{d t^{n}}\left(e^{-t^{2}}\right) .
\end{aligned}
$$

In particular, the normalized fundamental mode is given by

$$
\psi_{0}(t):=\sqrt[4]{\frac{v_{0}}{\pi}} e^{-v_{0}\left(t^{2} / 2\right)}
$$

Remark 3.1. An important property of the Hermite polynomials $H_{n}$ is the following orthogonality relation:

$$
\int_{\mathbb{R}} t^{k} e^{-t^{2}} H_{n}(t) \mathrm{d} t=0, \quad k=0,1, \ldots, n-1,
$$


Proof of Theorem 1.9. We divide the proof into four steps. In Step 1, we describe the asymptotic behavior of the first eigenpair of the cell problem. In Step 2, we prove a claim used in Step 1. In Step 3, we establish the asymptotic expansion (1.14) of the eigenvalues of problem (1.6) and, in Step 4, we prove that $v_{\varepsilon, k}^{\tau} \rightarrow 0$, as $\varepsilon \rightarrow 0^{+}$.

STEP 1. We prove that the asymptotic expansion of the first eigenpair $\left(\mu_{\varepsilon, 0}^{\tau}, \phi_{\varepsilon, 0}^{\tau}\right)$ of the cell problem (1.7), with $\tau>1$, takes the form, for $i \in \mathbb{N}_{0}, i>1$, and $\varepsilon<\varepsilon_{0}$ :

$$
\begin{gathered}
\mu_{\varepsilon, 0}^{\tau}=b_{\min } \rho_{0}+\varepsilon^{\tau-1} \nu_{0}+\varepsilon^{3(\tau-1) / 2} \mu_{3}+\cdots+\varepsilon^{(i+2)(\tau-1) / 2} \mu_{i+2}+r_{\varepsilon, i}^{\tau}, \\
\phi_{\varepsilon, 0}^{\tau}(y)=\varepsilon^{-(\tau-1) / 4}\left[\psi_{0}\left(\frac{y-y_{0}}{\sqrt{\varepsilon^{\tau-1}}}\right)+\varepsilon^{(\tau-1) / 2} \varphi_{1}\left(\frac{y-y_{0}}{\sqrt{\varepsilon^{\tau-1}}}\right)+\cdots\right. \\
\left.+\varepsilon^{i(\tau-1) / 2} \varphi_{i}\left(\frac{y-y_{0}}{\sqrt{\varepsilon^{\tau-1}}}\right)\right] \varsigma_{\varepsilon}(y)+R_{\varepsilon, i}^{\tau},
\end{gathered}
$$

where $\mu_{j}, j \in\{3, \ldots, i+2\}$, and $\phi_{j}, j \in\{1, \ldots, i\}$, are well-determined constants and functions, $\varsigma_{\varepsilon}$ is a convenient cut-off function in $Y$, and

$$
\max \left\{\left|r_{\varepsilon, i}^{\tau}\right|,\left\|R_{\varepsilon, i}^{\tau}\right\|_{L^{2}(Y)}\right\} \leq c \varepsilon^{(i+1)(\tau-1) / 2} .
$$

The proof is based on the asymptotic expansion techniques. We detail the proof for $i=1$ and then explain how to extend it for other eigenpairs.

Recalling problem (1.7), where $\phi_{\varepsilon}^{\tau}$ is determined up to a multiplicative constant,

$$
\left\{\begin{array}{l}
-\varepsilon^{2(\tau-1)}\left(a\left(\phi_{\varepsilon}^{\tau}\right)^{\prime}\right)^{\prime}+b \rho_{0} \phi_{\varepsilon}^{\tau}=\mu_{\varepsilon}^{\tau} \phi_{\varepsilon}^{\tau}, \quad \text { a.e. in } Y, \\
\phi_{\varepsilon}^{\tau} \in H_{\#}^{1}(Y),
\end{array}\right.
$$

we remark that it is well known (see [13], [14]) that $\mu_{\varepsilon, 0}^{\tau}-b_{\min } \rho_{0}$ behaves like $\varepsilon^{(\tau-1)}$. Also, from (3.2) and (3.3) we see that, when the coefficient $v_{0}$ of the harmonic oscillator is of order $1 / \varepsilon^{(\tau-1)}$, then the corresponding principal eigenmode $\psi_{0}$ behaves like a function of the argument $\left(t / \sqrt{\varepsilon^{\tau-1}}\right)$, and the normalization constant is of order $\sqrt[4]{\varepsilon^{\tau-1}}$.

Those facts justify the following change of variables, where we omit the fixed index $\tau$ :

$$
\begin{aligned}
& \epsilon=\sqrt{\varepsilon^{\tau-1}}, \quad t=\frac{y-y_{0}}{\epsilon}, \quad \mu_{\epsilon, 0}=\mu_{\varepsilon, 0}^{\tau}, \\
& \varphi_{\epsilon}(t)=\phi_{\varepsilon}^{\tau}\left(y_{0}+\epsilon t\right), \quad t \in Y_{\epsilon}:=\left[\frac{-y_{0}}{\epsilon}, \frac{1-y_{0}}{\epsilon}\right] .
\end{aligned}
$$


Without loss of generality we assume that $y_{0}$ is an interior point of $Y$ so that $y_{0}>0$ and $1-y_{0}>0$. Then, equation (1.7) takes the form:

$$
\left\{\begin{array}{l}
-\epsilon^{2}\left(a\left(y_{0}+\epsilon t\right)\left(\varphi_{\epsilon}\right)^{\prime}\right)^{\prime}+b\left(y_{0}+\epsilon t\right) \rho_{0} \varphi_{\epsilon}=\mu_{\epsilon} \varphi_{\epsilon}, \quad \text { a.e. in } Y_{\epsilon}, \\
\varphi_{\epsilon} \in H_{\#}^{1}\left(Y_{\epsilon}\right)
\end{array}\right.
$$

We assume the following expansions for the principal eigenpair of (3.8):

$$
\begin{aligned}
& \mu_{\epsilon, 0}=b_{\min } \rho_{0}+\epsilon^{2} \mu_{2}+\epsilon^{3} \mu_{3}+\cdots+\epsilon^{i+2} \mu_{i+2}+\cdots \\
& \varphi_{\epsilon, 0}(t)=\varphi_{0}(t)+\epsilon \varphi_{1}(t)+\epsilon^{2} \varphi_{2}(t)+\cdots+\epsilon^{i} \varphi_{i}(t)+\cdots
\end{aligned}
$$

and write down the following Taylor series:

$$
\begin{aligned}
a\left(y_{0}+\epsilon t\right)= & a\left(y_{0}\right)+\epsilon a^{\prime}\left(y_{0}\right) t+\epsilon^{2} \frac{a^{\prime \prime}\left(y_{0}\right)}{2} t^{2}+\cdots+\epsilon^{i+1} \frac{a^{(i+1)}\left(y_{0}\right)}{(i+1) !} t^{i+1} \\
& +\epsilon^{i+1} \frac{a^{(i+2)}(\theta)}{(i+2) !} t^{i+2}, \\
b\left(y_{0}+\epsilon t\right)= & b_{\min }+\epsilon^{2} \frac{b^{\prime \prime}\left(y_{0}\right)}{2} t^{2}+\epsilon^{3} \frac{b^{\prime \prime \prime}\left(y_{0}\right)}{3 !} t^{3}+\cdots+\epsilon^{i+1} \frac{b^{(i-1)}\left(y_{0}\right)}{(i+1) !} t^{i+1} \\
& +\epsilon^{i+2} \frac{b^{(i+2)}(\xi)}{(i+2) !} t^{i+2}
\end{aligned}
$$

where $\theta, \xi \in\left(y_{0}, y_{0}+\epsilon t\right)$. Then, substituting the above expansions in (3.8), collecting power like terms in the resulting equation, and taking the terms of order $\epsilon^{2}$ we conclude that $\varphi_{0}$ should satisfy

$$
-a\left(y_{0}\right) \varphi_{0}^{\prime \prime}+\frac{b^{\prime \prime}\left(y_{0}\right) \rho_{0}}{2} t^{2} \varphi_{0}=\mu_{2} \varphi_{0}, \quad \text { a.e. in } \mathbb{R} .
$$

So (see (3.2) and (3.3)), $\mu_{2}$ coincides with the first normalized eigenvalue of problem (3.9), more precisely

$$
\mu_{2}=v_{0}:=\sqrt{\frac{a\left(y_{0}\right) b^{\prime \prime}\left(y_{0}\right) \rho_{0}}{2}}
$$

and we choose

$$
\varphi_{0}=\psi_{0}:=\sqrt[4]{\frac{c\left(y_{0}\right)}{\pi}} e^{-c\left(y_{0}\right)\left(t^{2} / 2\right)} \quad \text { with } c\left(y_{0}\right):=\frac{v_{0}}{a\left(y_{0}\right)}=\sqrt{\frac{b^{\prime \prime}\left(y_{0}\right) \rho_{0}}{2 a\left(y_{0}\right)}}
$$


Collecting terms of order $\epsilon^{3}$, we obtain that $\varphi_{1}$ satisfies

$$
-a\left(y_{0}\right) \varphi_{1}^{\prime \prime}+\frac{b^{\prime \prime}\left(y_{0}\right) \rho_{0}}{2} t^{2} \varphi_{1}-v_{0} \varphi_{1}=a^{\prime}\left(y_{0}\right) \psi_{0}^{\prime}+a^{\prime}\left(y_{0}\right) t \psi_{0}^{\prime \prime}+\mu_{3} \psi_{0}
$$

a.e. in $\mathbb{R}$. Using the compatibility conditions given by the Fredholm alternative, we determine

$$
\mu_{3}:=a^{\prime}\left(y_{0}\right) \int_{\mathbb{R}} t\left|\psi_{0}^{\prime}\right|^{2} \mathrm{~d} t
$$

so that problem (3.12) has a unique solution $\varphi_{1}$ orthogonal to $\psi_{0}$.

Remark 3.2. We recall that the solution $\varphi_{1}$ of problem (3.13) can be represented by the series (see [7] for details)

$$
\varphi_{1}=\sum_{n \geq 1} \frac{\left(g, \psi_{n}\right)_{H}}{v_{n}-v_{0}} \psi_{n}
$$

where $g=a^{\prime}\left(y_{0}\right) \psi_{0}^{\prime}+a^{\prime}\left(y_{0}\right) t \psi_{0}^{\prime \prime}+\mu_{3} \psi_{0}$ and $(\cdot, \cdot)_{H}$ stands for the inner product in $H=L^{2}(\mathbb{R})$. Since $g$ can be written as a finite sum of terms of the form $c t^{k} \psi_{0}(t)$, in view of Remark 3.1 the sum in (3.14) turns out to be finite and, consequently, $\varphi_{1}(t)$ is also a finite linear combination of the terms $t^{k} \psi_{0}(t)$.

Repeating the described procedure, we determine the coefficients $\mu_{j}$ up to the order $(i+2)((3.10)$ and $(3.13)$ for $i=1)$ and $\varphi_{j}$, orthogonal to $\psi_{0}$, up to order $i$ ((3.9) and (3.12) for $i=1)$.

We now prove estimates (3.4)-(3.6), using Proposition 2.4.

Considering, for fixed $\epsilon, H=L_{\#}^{2}(Y)$ and $\mathscr{A}_{\epsilon} \phi:=-\epsilon^{4}\left(a(y) \phi^{\prime}\right)^{\prime}+b(y) \rho_{0} \phi$, $\phi \in H_{\#}^{1}(Y)$, we apply Proposition 2.4 to the operator $L_{\epsilon}: H \mapsto H$ that maps a function $g \in H$ to the unique solution of equation $\mathscr{A}_{\epsilon} \phi=g$. We define

$$
\tilde{w}_{\epsilon}(y):=\left[\psi_{0}\left(\frac{y-y_{0}}{\epsilon}\right)+\epsilon \varphi_{1}\left(\frac{y-y_{0}}{\epsilon}\right)+\cdots+\epsilon^{i} \varphi_{i}\left(\frac{y-y_{0}}{\epsilon}\right)\right] \varsigma(y),
$$

$f_{\epsilon}:=\mathscr{A}_{\epsilon} \tilde{w}_{\epsilon}$, and

$$
\tilde{\mu}_{\epsilon}:=b_{\min } \rho_{0}+\epsilon^{2} v_{0}+\epsilon^{3} \mu_{3}+\cdots+\epsilon^{i+2} \mu_{i+2},
$$

where $\varsigma \in C_{0}^{\infty}(0,1)$ is a cut-off function such that $0 \leq \varsigma \leq 1$, and $\varsigma(y)=1$ in a neighbourhood of $y_{0}$. 
Since $L_{\epsilon} f_{\epsilon}-\tilde{\mu}_{\epsilon}^{-1} f_{\epsilon}=\tilde{\mu}_{\epsilon}^{-1}\left(\tilde{\mu}_{\epsilon} \tilde{w}_{\epsilon}-\mathscr{A}_{\epsilon} \tilde{w}_{\epsilon}\right)$, using the definitions of $\left\{\mu_{j}\right\}_{j=3, \ldots, i+2}$ and $\left\{\varphi_{j}\right\}_{j=1, \ldots, i}$, the finite Taylor expansions of $a$ and $b$, and the fact that (see Remark 3.2), all the solutions $\varphi_{j}$ and its derivatives are finite sums of terms of the form $P(t) e^{-t^{2} / 2}$, where $P(t)$ are polynomials, we conclude that, for $\epsilon$ small enough, there exists a constant $C$, independent of $\epsilon$ (eventually different from line to line), such that

$$
\left\|L_{\epsilon} f_{\epsilon}-\tilde{\mu}_{\epsilon}^{-1} f_{\epsilon}\right\|_{H} \leq C \epsilon^{i+(3 / 2)} .
$$

This implies, by Proposition 2.4, the existence of an eigenvalue $\mu_{\epsilon, n}^{-1}$ of $L_{\epsilon}$ satisfying

$$
\left|\mu_{\epsilon, n}^{-1}-\tilde{\mu}_{\epsilon}^{-1}\right| \leq C\left\|f_{\epsilon}\right\|_{H}^{-1} \epsilon^{i+(3 / 2)} .
$$

Notice that $n$ might depend on $\epsilon$.

Since $\left\|f_{\epsilon}\right\|_{H} \geq b_{\min } \rho_{0}\left\|\tilde{w}_{\epsilon}\right\|_{H} \sim \sqrt{\epsilon}$, from (3.15) we conclude that

$$
\left|\mu_{\epsilon, n}^{-1}-\tilde{\mu}_{\epsilon}^{-1}\right| \leq C \epsilon^{i+1} .
$$

Because $\tilde{\mu}_{\epsilon}^{-1}$ is uniformly bounded away from zero, we obtain

$$
\left|\mu_{\epsilon, n}-\tilde{\mu}_{\epsilon}\right| \leq C \epsilon^{i+1} .
$$

Consequently, defining $v_{\epsilon, n}:=\frac{\mu_{\epsilon, n}-b_{\min } \rho_{0}}{\epsilon^{2}}$, we get that, for a certain $n$,

$$
\left|v_{\epsilon, n}-\left(v_{0}+\epsilon \mu_{3}+\cdots+\epsilon^{i} \mu_{i}\right)\right| \leq C \epsilon^{i-1} .
$$

We claim that for fixed $n$, as $\epsilon$ goes to zero, $v_{\epsilon, n}$ converges to the eigenvalue $v_{n}$ of problem (1.13). This statement is justified below, in Step 2. Then, for $\epsilon$ small enough, formulas (3.16)-(3.18) hold with $n=0$, that is

$$
\left|\mu_{\epsilon, 0}-\tilde{\mu}_{\epsilon}\right| \leq C \epsilon^{i+1} .
$$

Proposition 2.4 also yields the existence, for $\epsilon$ small enough, of an eigenfunction $w_{\epsilon, 0}$ associated to $\mu_{\epsilon, 0}$, having the same norm as $\tilde{w}_{\epsilon}$ and satisfying:

$$
\left\|\mathscr{A}_{\epsilon} w_{\epsilon, 0}-\mathscr{A}_{\epsilon} \tilde{\mathfrak{W}}_{\epsilon}\right\|_{H} \leq C \epsilon^{i+(3 / 2)} .
$$

Since $\left\|\tilde{w}_{\epsilon}\right\|_{H} \sim \sqrt{\epsilon}$ and, for a constant $C$ independent of $\epsilon$,

$$
\left\|w_{\epsilon, 0}-\tilde{w}_{\epsilon}\right\|_{H} \leq C\left\|\mathscr{A}_{\epsilon} w_{\epsilon, 0}-\mathscr{A}_{\epsilon} \tilde{w}_{\epsilon}\right\|_{H},
$$


we conclude from (3.20) that

$$
\left\|\phi_{\epsilon, 0}-\frac{1}{\sqrt{\epsilon}} \tilde{w}_{\epsilon}\right\|_{H} \leq C \epsilon^{i+1}
$$

From the definitions of $\tilde{w}_{\epsilon}$ and $\tilde{\mu}_{\epsilon}$, from (3.19) and (3.21), together with (3.7), we conclude estimates (3.4)-(3.6), which completes Step 1.

STEP 2. We prove that, for fixed $n, v_{\epsilon, n}$ converges, as $\epsilon$ goes to zero, to the eigenvalue $v_{n}$ of the harmonic oscillator (1.13). We present a proof for $n=0$. For the higher order eigenpairs the arguments are similar. In fact, we only need this statement for $n=0$ and $n=1$.

(i) We prove that $\liminf _{\epsilon \rightarrow 0^{+}} v_{\epsilon, 0} \geq v_{0}$. Suppose, up to restricting $\epsilon$ to a fixed sequence converging to zero, that $\liminf _{\epsilon \rightarrow 0^{+}} v_{\epsilon, 0}=\lim _{\epsilon \rightarrow 0^{+}} v_{\epsilon, 0}$ and that the limit is finite (otherwise (i) is immediate). Recalling (3.7), we have

$$
\begin{aligned}
v_{\epsilon, 0}= & \frac{\mu_{\epsilon, 0}-b_{\min } \rho_{0}}{\epsilon^{2}}=\inf _{\substack{\phi \in H_{\#}^{1}(Y) \\
\|\phi\|_{L^{2}(Y)}=1}} \int_{Y}\left(\epsilon^{2} a\left|\phi^{\prime}\right|^{2}+\frac{\left(b-b_{\min }\right) \rho_{0}}{\epsilon^{2}} \phi^{2}\right) \mathrm{d} y \\
= & \inf _{\substack{\varphi \in H_{\#}^{1}\left(Y_{\epsilon}\right) \\
\|\varphi\|_{L^{2}\left(Y_{\epsilon}\right)}}} \int_{Y_{\epsilon}}\left(a\left(y_{0}+\epsilon t\right)\left|\varphi^{\prime}\right|^{2}+\frac{\left(b\left(y_{0}+\epsilon t\right)-b_{\min }\right) \rho_{0}}{\epsilon^{2}} \varphi^{2}\right) \mathrm{d} t .
\end{aligned}
$$

Define

$$
G_{\epsilon}(\varphi):=\int_{Y_{\epsilon}}\left(a\left(y_{0}+\epsilon t\right)\left|\varphi^{\prime}\right|^{2}+\frac{\left(b\left(y_{0}+\epsilon t\right)-b_{\min }\right) \rho_{0}}{\epsilon^{2}} \varphi^{2}\right) \mathrm{d} t .
$$

For each $\epsilon$, let $\varphi_{\epsilon} \in H_{\#}^{1}\left(Y_{\epsilon}\right)$ be such that $\left\|\varphi_{\epsilon}\right\|_{L^{2}\left(Y_{\epsilon}\right)}=1$ and $v_{\epsilon, 0}>G_{\epsilon}\left(\varphi_{\epsilon}\right)-\epsilon$. Letting $\varphi_{\epsilon}=0$ for $y \in \mathbb{R} \backslash Y_{\epsilon}$, we extend $\varphi_{\epsilon}$ from $Y_{\epsilon}$ to the whole $\mathbb{R}$. For the extended function we keep the same name $\varphi_{\epsilon}$. Since $b$ attains a global minimum $b_{\min }$ at a unique point $y_{0} \in Y$, there exists $\eta_{0}>0$ such that $0<1 / \eta_{0}<\left(b(y)-b\left(y_{0}\right)\right) /$ $\left(y-y_{0}\right)<\eta_{0}$ for all $y \in Y$ and, consequently,

$$
t^{2} \eta_{0}>\frac{b\left(y_{0}+\epsilon t\right)-b_{\min }}{\epsilon^{2}}>\frac{t^{2}}{\eta_{0}}
$$

We have, then, the following uniform in $\epsilon$ bounds:

$$
0<G_{\epsilon}\left(\varphi_{\epsilon}\right) \leq C, \quad \int_{Y_{\epsilon}}\left|\varphi_{\epsilon}^{\prime}\right|^{2} \mathrm{~d} t \leq C, \quad \int_{Y_{\epsilon}} t^{2} \varphi_{\epsilon}^{2} \mathrm{~d} t \leq C, \quad \int_{\mathbb{R}} \varphi_{\epsilon}^{2} \mathrm{~d} t=1
$$


Up to extracting a subsequence of $\left\{\varphi_{\epsilon}\right\}$, we may assume that $\varphi_{\epsilon} \rightarrow \varphi$ weakly in $L^{2}(\mathbb{R})$. Also, for every finite $\eta>0,\left\{\varphi_{\epsilon}\right\}$ is bounded in $H^{1}(-\eta, \eta)$ so that by Rellich-Kondrachov Theorem, it holds

$$
\lim _{\epsilon \rightarrow 0^{+}} \int_{(-\eta, \eta)}\left|\varphi_{\epsilon}\right|^{2} \mathrm{~d} t=\int_{(-\eta, \eta)}|\varphi|^{2} \mathrm{~d} t
$$

Using the lower semicontinuity of the norm with respect to the weak topology, the convergence (3.23), and exploiting the third inequality in (3.22), we obtain, since $\varphi_{\epsilon}=0$ outside $Y_{\epsilon}$,

$$
\int_{\mathbb{R}}|\varphi|^{2} \mathrm{~d} t \leq \liminf _{\epsilon \rightarrow 0^{+}} \int_{Y_{\epsilon}}\left|\varphi_{\epsilon}\right|^{2} \mathrm{~d} t \leq \limsup _{\epsilon \rightarrow 0^{+}} \int_{Y_{\epsilon}}\left|\varphi_{\epsilon}\right|^{2} \mathrm{~d} t \leq \int_{|t| \leq \eta}|\varphi|^{2} \mathrm{~d} t+\frac{C}{\eta^{2}},
$$

from which follows the strong convergence of $\varphi_{\epsilon}$ in $L^{2}(\mathbb{R})$ by sending $\eta$ to infinity.

Then,

$$
\|\varphi\|_{L^{2}(\mathbb{R})}=1, \quad \varphi_{\epsilon}^{\prime} \mathbf{1}_{Y_{\epsilon}} \rightarrow \varphi^{\prime} \text { in } L_{l o c}^{2}(\mathbb{R})
$$

where $\mathbf{1}_{Y_{\epsilon}}$ stands for the characteristic function of $Y_{\epsilon}$. Furthermore, both sequences $a\left(y_{0}+\epsilon t\right)$ and $\frac{b\left(y_{0}+\epsilon t\right)-b_{\min }}{\epsilon^{2}}$ converge uniformly on compacts to $a\left(y_{0}\right)$ and $\frac{b^{\prime \prime}\left(y_{0}\right)}{2} t^{2}$, respectively. Consequently, for $\theta \in \mathscr{D}(\mathbb{R}), 0 \leq \theta \leq 1$, using the lower semicontinuity of the norm with respect to the weak convergence and Fatou's Lemma, we obtain

$$
\begin{aligned}
\liminf _{\epsilon \rightarrow 0^{+}} G_{\epsilon}\left(\varphi_{\epsilon}\right) & \geq \liminf _{\epsilon \rightarrow 0^{+}} \int_{\mathbb{R}} \theta\left(a\left(y_{0}+\epsilon t\right)\left|\varphi_{\epsilon}^{\prime}\right|^{2}+\frac{\left(b\left(y_{0}+\epsilon t\right)-b_{\min }\right) \rho_{0}}{\epsilon^{2}} \varphi_{\epsilon}^{2}\right) \mathrm{d} t \\
& \geq \int_{\mathbb{R}} \theta\left(a\left(y_{0}\right)\left|\varphi^{\prime}\right|^{2}+\frac{b^{\prime \prime}\left(y_{0}\right)}{2} \rho_{0} t^{2} \varphi^{2}\right) \mathrm{d} t .
\end{aligned}
$$

Letting $\theta \rightarrow 1$ we get

$$
\lim _{\epsilon \rightarrow 0^{+}} v_{\epsilon, 0} \geq \liminf _{\epsilon \rightarrow 0^{+}} G_{\epsilon}\left(\varphi_{\epsilon}\right) \geq \int_{\mathbb{R}}\left(a\left(y_{0}\right)\left|\varphi^{\prime}\right|^{2}+\frac{b^{\prime \prime}\left(y_{0}\right)}{2} \rho_{0} t^{2} \varphi^{2}\right) \mathrm{d} t \geq v_{0},
$$

which concludes the proof of (i).

(ii) We prove that $\limsup _{\epsilon \rightarrow 0^{+}} v_{\epsilon, 0} \leq v_{0}$. It is enough to prove the existence of a sequence $\left\{\varphi_{\epsilon}\right\}$ in $H_{\#}^{1}\left(Y_{\epsilon}\right)$ such that $\left\|\varphi_{\epsilon}\right\|_{L^{2}\left(Y_{\epsilon}\right)}=1$ and $G_{\epsilon}\left(\varphi_{\epsilon}\right) \rightarrow v_{0}$.

Defining

$$
\varphi_{\epsilon}(t):=\frac{\psi_{0}(t) \varsigma\left(y_{0}+\epsilon t\right)}{\left\|\psi_{0} \varsigma\left(y_{0}+\epsilon \cdot\right)\right\|_{L^{2}\left(Y_{\epsilon}\right)}}
$$


where $\psi_{0}$ is the first eigenmode of the harmonic oscillator and $\varsigma \in C_{0}^{\infty}(0,1)$ is the cut-off function such that $0 \leq \varsigma \leq 1$, and $\varsigma(y)=1$ in a neighbourhood of $y_{0}$, we obtain, using the definitions of $\psi_{0}$ and $\varsigma$,

$$
\begin{aligned}
\lim _{\epsilon \rightarrow 0^{+}} & G_{\epsilon}\left(\varphi_{\epsilon}\right) \\
& =\lim _{\epsilon \rightarrow 0^{+}}\left(\frac{1}{1+o(\epsilon)}\left[\int_{\mathbb{R}}\left(a\left(y_{0}+\epsilon t\right)\left|\psi_{0}^{\prime}\right|^{2}+\frac{\left(b\left(y_{0}+\epsilon t\right)-b_{\min }\right) \rho_{0}}{\epsilon^{2}} \psi_{0}^{2}\right) \mathrm{d} t+o(\epsilon)\right]\right) \\
& =\int_{\mathbb{R}}\left(a\left(y_{0}\right)\left|\psi_{0}^{\prime}\right|^{2}+\frac{b^{\prime \prime}\left(y_{0}\right)}{2} \rho_{0} t^{2} \psi_{0}^{2}\right) \mathrm{d} t=v_{0} .
\end{aligned}
$$

STEP 3. The asymptotic expansion (1.14) is an immediate consequence of Propositions 2.1 and 2.2, applied to the rescaled problem (1.6) with $\delta=\varepsilon^{\tau}$, of Remark 2.3, which yields (see 2.3)

$$
\lambda_{\varepsilon, k}=\lambda_{\varepsilon, k}^{(0)}=\frac{\mu_{\varepsilon, 0}^{\tau}}{\varepsilon^{2 \tau}}+v_{\varepsilon, k}^{\tau}
$$

and of formula 3.4, obtained in Step 1.

STEP 4. It remains to prove that $v_{\varepsilon, k}^{\tau} \rightarrow 0$, as $\varepsilon \rightarrow 0^{+}$, for any $k=0,1, \ldots$ In fact, $v_{\varepsilon, k}^{\tau}$ converge to zero exponentially. By the max-min principle we have

$$
v_{\varepsilon, k}^{\tau}:=\inf _{S_{k}} \max _{\substack{\left.\psi \in S_{k} \\ \| \phi_{\varepsilon, 0}^{\tau}, \frac{(-)}{\varepsilon}\right) \psi_{L^{2}(I)}=1}}\left\{\int_{I}\left|\phi_{\varepsilon, 0}^{\tau}\left(\frac{x_{3}}{\varepsilon}\right)\right|^{2} a_{\varepsilon}\left|\psi^{\prime}\right|^{2} \mathrm{~d} x_{3}\right\},
$$

where the infimum is taken over all $(k+1)$-dimensional subspaces $S_{k}$ of $H$.

For a fixed $k \geq 0$ let $\xi \in C_{0}^{\infty}(0, L /(k+1))$ be a positive function such that $\|\xi\|_{L^{2}(0, L)}=1$. Denote by $\xi_{\varepsilon}$ a piece-wise linear approximation of this function constructed in the following way. In the $(\varepsilon / 10)$-neighborhoods of the minimum points of $b\left(\frac{\dot{\bar{\varepsilon}}}{\varepsilon}\right.$, which are $\varepsilon y_{0}, \varepsilon y_{0}+\varepsilon, \ldots$, the derivative of $\xi_{\varepsilon}$ is equal to 0 . In the complement to these intervals, $\left|\xi_{\varepsilon}^{\prime}(t)\right| \leq C$. Moreover, $\left|\xi-\xi_{\varepsilon}\right| \leq C \varepsilon$. Considering the structure of $\phi_{\varepsilon, 0}^{\tau}(y)$, one can easily check that

$$
\left\|\phi_{\varepsilon, 0}^{\tau}\left(\frac{\dot{q}}{\varepsilon}\right) \xi_{\varepsilon}(\cdot)\right\|_{L^{2}(I)}=1+o(1)
$$

where $o(1)$ tends to zero, as $\varepsilon \rightarrow 0^{+}$, and

$$
\left\{\int_{I}\left|\phi_{\varepsilon, 0}^{\tau}\left(\frac{x_{3}}{\varepsilon}\right)\right|^{2} a_{\varepsilon}\left|\xi_{\varepsilon}^{\prime}\right|^{2} \mathrm{~d} x_{3}\right\} \leq C \varepsilon^{(1-\tau) / 4} \exp \left(-\frac{c\left(y_{0}\right)}{200} \varepsilon^{1-\tau}\right),
$$


where $c\left(y_{0}\right)=v_{0} / a\left(y_{0}\right)$ has been defined in (3.11). Shifting the argument of $\xi_{\varepsilon}$ by $L /(k+1), 2 L /(k+1)$, etc., we arrive at an orthogonal family of $k+1$ functions which satisfy (3.24) and (3.25). This yields the desired statement.

\section{References}

[1] G. Allaire and F. Malige, Analyse asymptotique spectrale d'un problème de diffusion neutronique. C. R. Acad. Sci. Paris Sér. I Math. 324, 8 (1997), 939-944.

[2] D. Borisov and P. Freitas, Asymptotics of Dirichlet eigenvalues and eigenfunctions of the Laplacian on thin domains in $\mathbb{R}^{d}$. J. Funct. Anal. 258, 3 (2010), 893-912.

[3] G. Bouchitte, M. L. Mascarenhas, and L. Trabucho, On the curvature and torsion effects in one dimensional waveguides. ESAIM Control Optim. Calc. Var. 13, 4 (2007), 793-808 (electronic).

[4] G. Bouchitte, M. L. Mascarenhas, and L. Trabucho, Thin waveguides with robin boundary conditions. J. Math. Phys. 53, 12 (December 2012).

[5] G. Cao, Nanostructures and Nanomaterials, Synthesis, Properties and Applications. Imperial College Press, London, 2004.

[6] B. Chenaud, P. Duclos, P. Freitas, and D. Krejčiřík, Geometrically induced discrete spectrum in curved tubes. Differential Geom. Appl. 23, 2 (2005), 95-105.

[7] R. Dautray and J.-L. Lions, Analyse mathématique et calcul numérique pour les sciences et les techniques. Vol. 5. Masson, Paris, 1988.

[8] P. Duclos and P. Exner, Curvature-induced bound states in quantum waveguides in two and three dimensions. Rev. Math. Phys. 7, 1 (1995), 73-102.

[9] R. Ferreira and M. L. Mascarenhas, Waves in a thin and periodically oscillating medium. C. R. Math. Acad. Sci. Paris 346, 9-10 (2008), 579-584.

[10] R. Ferreira, M. L. Mascarenhas, and A. Piatnitski, Spectral analysis in a thin domain with periodically oscillating characteristics. ESAIM Control Optim. Calc. Var. 18, 2 (2012), 427-451.

[11] L. Friedlander and M. Solomyak, On the spectrum of narrow periodic waveguides. Russ. J. Math. Phys. 15, 2 (2008), 238-242.

[12] D. Gilbarg and N. Trudinger, Elliptic partial differential equations of second order. Springer-Verlag, Berlin-New York, 1977.

[13] S. Kozlov and A. Piatnitski, Effective diffusion for a parabolic operator with periodic potential. SIAM J. Appl. Math. 53, 2 (1993), 401-418.

[14] S. Kozlov and A. Piatnitski, Degeneration of effective diffusion in the presence of periodic potential. Ann. Inst. H. Poincaré Probab. Statist. 32, 5 (1996), 571-587.

[15] C. Kreisbeck and M. L. Mascarenhas, Asymptotic spectral analysis in semiconductor nanowire heterostructures. Appl. Anal. 94, 6 (2015), 1153-1191.

[16] D. Krejčiř́ik and H. Šediváková, The effective Hamiltonian in curved quantum waveguides under mild regularity assumptions. Rev. Math. Phys. 24, 7 (2012), 1250018, 39. 
[17] L. J. Lauhon, M. S. Gudiksen, and C. M. Lieber, Semiconductor nanowire heterostructures. Philos. Trans. R. Soc. Lond. Ser. A-Math. Phys. Eng. Sci. 362, 1819 (2004), 1247-1260.

[18] L. J. Lauhon, M. S. Gudiksen, C. L. Wang, and C. M. Lieber, Epitaxial core-shell and core-multishell nanowire heterostructures. Nature 420, 6911 (2002), 57-61.

[19] L. C. Lew Yan Voon and M. Willatzen, Electron states in modulated nanowires. J. Appl. Phys. 93, 12 (2003), 9997-10000.

[20] O. A. Oleĭnik, A. S. Shamaev, and G. A. Yosifian, Mathematical problems in elasticity and homogenization, vol. 26 of Studies in Mathematics and its Applications. NorthHolland Publishing Co., Amsterdam, 1992.

[21] Y. Qu and X. Duan, One-dimensional homogeneous and heterogeneous nanowires for solar energy conversion. J. Mater. Chem. 22, 32 (2012), 16171-16181.

[22] M. Vanninathan, Homogenization of eigenvalue problems in perforated domains. Proc. Indian Acad. Sci. Math. Sci. 90, 3 (1981), 239-271.

[23] M. I. Višik and L. A. Ljusternik, Regular degeneration and boundary layer for linear differential equations with small parameter. Amer. Math. Soc. Transl. (2) 20 (1962), 239-364.

[24] H. Yan, H. S. Choe, S. Nam, Y. Hu, S. Das, J. F. Klemic, J. C. Ellenbogen, and C. M. Lieber, Programmable nanowire circuits for nanoprocessors. Nature 470, 7333 (2011), 240-244.

[25] K. Young, Position-dependent effective mass for inhomogeneous semiconductors. Phys. Rev. B 39, 18 (1989), 13434-13441.

Received September 17, 2014

R. Ferreira, CEMSE Division \& KAUST SRI, Uncertainty Quantification Center in Computational Science and Engineering, King Abdullah University of Science and Technology (KAUST), Thuwal 23955-6900, Saudi Arabia

E-mail: rita.ferreira@kaust.edu.sa

M. L. Mascarenhas, Departamento de Matemática \& C.M.A., F.C.T./U.N.L., Quinta da Torre, 2829-516 Caparica, Portugal

E-mail: mascar@fct.unl.pt

A. Piatnitski, Narvik University College, P.O. Box 385, 8505 Narvik, Norway \& P.N. Lebedev Physical Institute RAS, Leninski prospect 53, Moscow 119991, Russia

E-mail: andrey@sci.lebedev.ru 